\title{
Role of interleukin-6 gene polymorphisms in the development of prostate cancer
}

\author{
C.H. Chen, M. Gong, Q.T. Yi and J.H. Guo \\ Department of Urology, Shanghai Pudong Hospital, \\ Fudan University Pudong Medical Center, Huinan Town, Pudong, Shanghai, China \\ Corresponding author: M. Gong \\ E-mail: mingong_sph@163.com \\ Genet. Mol. Res. 14 (4): 13370-13374 (2015) \\ Received April 30, 2015 \\ Accepted August 18, 2015 \\ Published October 26, 2015 \\ DOI http://dx.doi.org/10.4238/2015.October.26.34
}

ABSTRACT. We investigated the role of two functional polymorphisms, IL6-174G>C (rs1800795) and IL-6-572C>G (rs1800796), in the development of prostate cancer. A total of 212 consecutive primary prostate cancer patients and 236 control subjects were recruited between May 2012 and May 2014. The IL-6-174G>C (rs1800795) and IL-6-572C>G (rs1800796) polymorphisms were assessed by polymerase chain reaction-restriction fragment length polymorphism. The genotype distributions of IL-6174G $>C$ (rs1800795) and IL-6-572C $>$ G (rs1800796) met the HardyWeinberg equilibrium in the controls. Unconditional logistic regression analyses showed that the GG genotype of IL-6-572C>G (rs1800796) was associated with an elevated risk of prostate cancer compared with the CC genotype, and the OR $(95 \% \mathrm{Cl})$ for the GG genotype was 2.06 (1.113.87). In conclusion, we suggest that the IL-6-572C>G (rs1800796) gene polymorphism influences the development of prostate cancer.

Key words: Interleukin-6; Polymorphism; Prostate cancer 


\section{INTRODUCTION}

Prostate cancer is the second most common cancer diagnosed in men, and the fourth most common cancer overall worldwide. It is estimated that 1.1 million men were diagnosed with prostate cancer in 2012 worldwide, which accounted for $15 \%$ of cancers in men (Jemal et al., 2008). About $70 \%$ of prostate cancer patients were diagnosed in the less developed regions (Jemal et al., 2008). Prostate cancer is complex and is caused by many environmental and lifestyle factors, such as ultraviolet irradiation, smoking, and diet (Grant, 2004). However, not all potential prostate cancer patients develop the disease, even when they have been exposed to such risk factors, which suggests that genetic variations may influence the development of prostate cancer.

Previous studies have shown that many oxidants produced by inflammatory cells may cause cellular or genomic damage, enhance cell proliferation, and stimulate angiogenesis (Hughes et al., 2005; Shen and Abate-Shen, 2010). Interleukin-6 (IL-6) is a multifunctional cytokine and is encoded by the IL6 gene, which has the chromosomal locus 7p21-14. It has been reported that IL-6 activates the signal transducer and activator of transcription (STAT) and mitogen-activated protein kinase (MAPK) signaling pathways, as well as transcript target genes, and also regulates cell proliferation and metabolism (Hughes et al., 2005; Culig and Puhr, 2012). Therefore, this cytokine could contribute to the initiation, development, and metastasis of prostate cancer. Several studies have shown an association between IL-6 gene polymorphisms and the risk of prostate cancer, but the results are inconclusive (Magalhães et al., 2013; Mandić et al., 2013; Mandal et al., 2014; Yang et al., 2014). In this study, we investigated the role of two functional polymorphisms, IL-6-174G>C (rs1800795) and IL-6-572C>G (rs1800796), in the development of prostate cancer.

\section{MATERIAL AND METHODS}

\section{Subjects}

A total of 212 consecutive primary prostate cancer patients and 236 control subjects were recruited from the Shanghai Pudong Hospital between May 2012 and May 2014. Initially, 238 prostate cancer patients were diagnosed and histopathologically confirmed by two pathologists. Patients who had received chemotherapy or radiotherapy before participating in this study, or patients who had suffered from an infectious disease or diabetes mellitus, were excluded. Ultimately, 212 patients were included in our study, and the participation rate was $89.08 \%$. A total of 264 controls were randomly selected from individuals who had undergone a routine health checkup during the same period, and the controls who had a history of cancer, infectious disease, or diabetes mellitus were excluded. Ultimately, 236 control subjects were recruited for analysis, with a participation rate of $89.39 \%$. The ethics committee of the Shanghai Pudong Hospital approved the study, and each patient gave their written consent.

\section{Sequencing analysis and genotyping}

Before the patients began their treatment, $2 \mathrm{~mL}$ peripheral blood was collected using ethylenediaminetetraacetic acid (EDTA)-anticoagulant tubes. The blood samples were stored at $-20^{\circ} \mathrm{C}$ until required. Genomic DNA was isolated from peripheral blood leukocytes using a TIANamp Blood DNA Kit (Tiangen Biotech, Beijing, China) according to the manufacturer instructions, and genomic DNA was stored at $-20^{\circ} \mathrm{C}$ until required. The IL-6-174G>C (rs1800795) and IL-6- 
572C>G (rs1800796) polymorphisms were assessed by polymerase chain reaction-restriction fragment length polymorphism (PCR-RFLP). The primers for IL-6-174G>C (rs1800795) and IL6-572C>G (rs1800796) were designed using Sequenom Assay Design 3.1 software. The primers for IL-6-174G>C were as follows: forward, 5'-AGAATCTTTGACGAGGTTTG-3' and reverse, 5'-GGCCATGATTGGAGGCCTTA-3'. The primers for IL-6-572G/C were as follows: forward, 5'-GCACGATTTAAGACCATGGC-3' and reverse, 5'-TCTGAGCCTCCTCGTGTTCTGG-3'. The PCR was performed using the following conditions: initial denaturation at $94^{\circ} \mathrm{C}$ for $5 \mathrm{~min}$; 35 cycles of denaturation at $94^{\circ} \mathrm{C}$ for $45 \mathrm{~s}$, annealing at $62^{\circ} \mathrm{C}$ for $60 \mathrm{~s}$, and extension at $72^{\circ} \mathrm{C}$ for $60 \mathrm{~s}$; and final extension at $72^{\circ} \mathrm{C}$ for $10 \mathrm{~min}$.

\section{Statistical analysis}

Continuous variables are expressed as mean \pm standard deviation (SD), and categorical variables are expressed as frequency (percentage) of study participants. The Student $t$-test was used to compare continuous variables and the $X^{2}$-test was used to compare categorical variables between patients and control subjects. Hardy-Weinberg equilibrium (HWE) among the controls was compared using the $X^{2}$-test. Unconditional logistic regression was conducted to assess the effects of IL-6-174G >C (rs1800795) and IL-6-572C>G (rs1800796) polymorphisms on the risk of prostate cancer, and the results are expressed as ORs and $95 \% \mathrm{Cls}$. All P values were two sided, and $P<0.05$ was considered statistically significant. All statistical analyses were conducted using the SPSS ${ }^{\circledR}$ statistical package, version 11.0 (SPSS Inc., Chicago, IL, USA) for Windows ${ }^{\circledR}$.

\section{RESULTS}

The demographic and clinical characteristics of the study subjects are shown in Table 1. As expected, no significant difference was identified between the prostate cancer patients and the control subjects in terms of age. Moreover, we did not find a significant association between the prostate cancer patients and the control subjects in terms of alcohol consumption, smoking tobacco, or history of cancer in the first relatives.

\begin{tabular}{|c|c|c|c|c|c|c|}
\hline Characteristics & Patients $N=212$ & $\%$ & Controls $\mathrm{N}=236$ & $\%$ & $x^{2}$ value & $P$ value \\
\hline Mean age, years & $64.56 \pm 9.50$ & $65.15 \pm 9.81$ & 0.65 & 0.26 & & \\
\hline \multicolumn{7}{|c|}{ Alcohol consumption } \\
\hline No & 80 & 37.74 & 105 & 44.49 & & \\
\hline Yes & 132 & 62.26 & 131 & 55.51 & 2.10 & 0.15 \\
\hline \multicolumn{7}{|l|}{ Smoking tobacco } \\
\hline No & 95 & 44.81 & 114 & 48.31 & & \\
\hline Yes & 117 & 55.19 & 122 & 51.69 & 0.55 & 0.46 \\
\hline \multicolumn{7}{|c|}{ History of cancer in the first relatives } \\
\hline No & 195 & 91.98 & 225 & 95.34 & & \\
\hline Yes & 17 & 8.02 & 11 & 4.66 & 2.15 & 0.14 \\
\hline \multicolumn{7}{|l|}{ Gleason score } \\
\hline$<7$ & 79 & 37.26 & & & & \\
\hline$\geq 7$ & 133 & 62.74 & & & & \\
\hline
\end{tabular}

The genotype distributions of IL-6-174G>C (rs1800795) and IL-6-572C>G (rs1800796) met the HWE in the controls, and the $P$ values for HWE were 0.27 and 0.85 , respectively (Table 2). Moreover, we found that the minor allele frequencies (MAF) of IL-6-174G>C (rs1800795) and IL- 
6-572C>G (rs1800796) were similar to MAFs in the National Center for Biotechnology Information SNP database (http://www.ncbi.nlm.nih.gov/snp/).

Table 2. Genotype frequencies of IL-6-174G>C (rs1800795) and IL-6-572C>G (rs1800796) polymorphisms in prostate cancer cases and controls.

\begin{tabular}{lccccc}
\hline Gene & SNP & Alleles & Hardy-Weinberg equilibrium & \multicolumn{2}{c}{ Minor allele frequency (MAF) } \\
\cline { 5 - 6 } & & & & In controls & In database \\
\hline IL-6-174G>C & rs1800795 & G/C & 0.27 & 0.186 & 0.185 \\
IL-6-572C>G & rs1800796 & C/G & 0.85 & 0.322 & 0.314 \\
\hline
\end{tabular}

Unconditional logistic regression analyses showed that subjects carrying the GG genotype of IL-6-572C>G (rs1800796) were at a higher risk of developing prostate cancer than those carrying the CC genotype. The OR $(95 \% \mathrm{Cl})$ for the GG genotype of IL-6-572C>G (rs1800796) was 2.06 (1.11-3.87) compared with the CC genotype (Table 3). However, we did not find any significant association between the IL-6-572C>G gene polymorphism and risk of prostate cancer.

\begin{tabular}{|c|c|c|c|c|c|c|}
\hline Gene & Patients & $\%$ & Controls & $\%$ & OR $(95 \% \mathrm{Cl})^{1}$ & $P$ value \\
\hline \multicolumn{7}{|c|}{ IL-6-174G $>C$} \\
\hline GG & 131 & 61.79 & 158 & 66.95 & 1.0 (Ref.) & - \\
\hline GC & 64 & 30.19 & 67 & 28.39 & $1.15(0.75-1.78)$ & 0.50 \\
\hline $\mathrm{CC}$ & 17 & 8.02 & 11 & 4.66 & $1.86(0.79-4.56)$ & 0.12 \\
\hline \multicolumn{7}{|c|}{ IL-6-572C>G } \\
\hline $\mathrm{CC}$ & 79 & 37.26 & 110 & 46.61 & 1.0 (Ref.) & - \\
\hline CG & 96 & 45.28 & 102 & 43.22 & $1.31(0.86-2.00)$ & 0.18 \\
\hline GG & 37 & 17.45 & 25 & 10.59 & $2.06(1.11-3.87)$ & 0.01 \\
\hline
\end{tabular}

\section{DISCUSSION}

Genetic susceptibility to disease has attracted increasing attention in recent years, and gene polymorphisms that are involved in various diseases are of particular interest. Previous studies have shown that a gene polymorphism encoding an inflammatory mediator can affect the plasma level and biological activity of the related inflammatory protein (Howell and Rose-Zerilli, 2007). Therefore, polymorphisms of cytokine genes can influence susceptibility to prostate cancer and disease severity (Yang et al., 2014). In this study, we report that the GG genotype of IL-6-572C>G (rs1800796) is associated with an elevated risk of prostate cancer compared with the $\mathrm{CC}$ genotype.

The authors of several previous studies have reported an association between IL- 6 gene polymorphisms and prostate cancer risk, but the results are inconsistent (Bao et al., 2008; Magalhães et al., 2013; Mandić et al., 2013; Mandal et al., 2014; Yang et al., 2014). Mandal et al. (2014) conducted a study in Caucasian subjects and found that the GG genotype of IL-6-174G>C was associated with increased risk of prostate cancer. Mandić et al. (2013) reported that IL-6174G >C gene polymorphisms influence susceptibility to prostate cancer. However, some studies have produced inconsistent results; Magalhães et al. (2013) conducted a case-control study to assess the role of IL-6-174G >C and IL-6-636G>C in the development of prostate cancer, and found no significant association between the IL-6-174G $>C$ polymorphism and increased risk of prostate cancer. Bao et al. (2008) reported that the GG+CG genotype of IL-6-174G>C was correlated with 
increased risk of prostate cancer compared with the CC genotype. The discrepancies in the abovementioned results may be caused by differences in case selection, ethnicities, sample size, and also by chance.

Three limitations should be considered in our study. First, cases and controls were selected from one hospital, which may not represent the general population. Second, owing to the rarity of prostate cancer, the sample size of patients in our study was relatively small, which may have limited the ability to identify statistically significant differences between the groups. Third, the development of prostate cancer may be influenced by many other genetic factors in addition to that of the IL-6 gene polymorphism. Therefore, further studies with a large sample size are needed to confirm the association.

In conclusion, we suggest that the IL-6-572C>G ( $\mathrm{rs} 1800796$ ) gene polymorphism influences the development of prostate cancer. Owing to the limitations of our study, further welldesigned, multicenter studies with a large sample size would be very helpful.

\section{Conflicts of interest}

The authors declare no conflict of interest.

\section{ACKNOWLEDGMENT}

Research supported by the Academic Leaders Training Program of Pudong Health Bureau of Shanghai (\#PWRd2014-06) and the Key Discipline Construction Project of Pudong Health Bureau of Shanghai (PWZx2014-04).

\section{REFERENCES}

Bao S, Yang W, Zhou S and Ye Z (2008). Relationship between single nucleotide polymorphisms in -174G/C and -634C/G promoter region of interleukin-6 and prostate cancer. J. Huazhong Univ. Sci. Technolog. Med. Sci. 28: 693-696.

Culig Z and Puhr M (2012). Interleukin-6: a multifunctional targetable cytokine in human prostate cancer. Mol. Cell. Endocrinol. 360: 52-58.

Grant WB (2004). A multicountry ecologic study of risk and risk reduction factors for prostate cancer mortality. Eur. Urol. 45: 271-279.

Howell WM and Rose-Zerilli MJ (2007). Cytokine gene polymorphisms, cancer susceptibility, and prognosis. J. Nutr. 137: 194S-199S.

Hughes C, Murphy A, Martin C, Sheils O, et al. (2005). Molecular pathology of prostate cancer. J. Clin. Pathol. 58: 673-684.

Jemal A, Siegel R, Ward E, Hao Y, et al. (2008). Cancer statistics, 2008. CA Cancer J. Clin. 58: 71-96.

Magalhães JF, Cortinhas AJ, Albuquerque CM, Baptista CS, et al. (2013). Interleukin-6 gene -174G>C and -636G>C promoter polymorphisms and prostate cancer risk. Mol. Biol. Rep. 40: 449-455.

Mandal S, Abebe F and Chaudhary J (2014). -174G/C polymorphism in the interleukin-6 promoter is differently associated with prostate cancer incidence depending on race. Genet. Mol. Res. 13: 139-151.

Mandić S, Sudarević B, Marczi S, Horvat V, et al. (2013). Interleukin-6 polymorphism and prostate cancer risk in population of Eastern Croatia. Coll. Antropol. 37: 907-911.

Shen MM and Abate-Shen C (2010). Molecular genetics of prostate cancer: new prospects for old challenges. Genes Dev. 24: 1967-2000.

Yang M, Li C and Li M (2014). Association of interleukin-6 (-174 G/C) polymorphism with the prostate cancer risk: A metaanalysis. Biomed. Rep. 2: 637-643. 